\title{
Human-centered Approach for Proper Functioning of LEED Criteria; A Case Study in Tehran
}

Seyed Hossein Zargar ${ }^{1 *}$, Fatemeh Dehghani Firouzabadi ${ }^{1}$, Mojtaba Zareie ${ }^{2}$, Saeid Khaghani ${ }^{1}$

${ }^{1}$ School of Architecture, Faculty of Fine Arts, University of Tehran, Tehran, Iran

${ }^{2}$ Dept of Architecture, Pars University, Tehran, Iran

* Corresponding author email: s.hossein.zargar@gmail.com

Received: 05 October 2019 / Revised: 04 November 2019 / Accepted: 05 November 2019 / Published: 10 November 2019

\begin{abstract}
Buildings are rated in five main categories according to the LEED standard to receive an environmentfriendly design certificate. Sustainability, water efficiency, material properties, energy consumption, and the quality of indoor spaces are main criteria which confirm the proper function of a building in terms of optimal energy consumption. However, the role of residents and behavioral aspects in the consumption of energy and the decent application of energy standards are neglected. It is beyond doubt that the residents' behavior is one of the most important factors in performance-based building design. Moreover, the individual mistakes, which most of the times cause energy dissipation, cannot be ignored. This research proposes "Integrated Interactive System" as a human-centered approach with the aim of minimizing energy consumption. A survey is conducted, and this system is evaluated as a case study in Tehran.
\end{abstract}

Keywords: LEED, Residents' Behavior, Energy Consumption, Sustainability, Human-centered Design

\section{Introduction}

In previous decades, the concept of sustainable development had turned into the center of attention by the international community and architectural engineers. Hundreds of international conferences and conventions have organized around the world with the aim of retrieving the balance lifecycle of the Earth. The general goal is to reduce the negative effects on the ecosystem by enforcing different rules and regulations. The Brundtland Commission, the Kyoto Protocol, and the Maastricht Treaty are only part of the international treatments targeting the goal of finding best scenarios in the realm of energy consumption. Statistics shows that buildings themselves are great energy consumers (Janda, 2011). Different research indicates that the increasing advancement of technology and financial investments do not guarantee a low or zero energy consumption of the building. In fact, human factors play an important role in this issue, and despite the improvement of human understanding of these criteria in recent years, it is often overlooked in building design (D’Oca, 2018). Some studies point out the big gap between the actual and estimated performance of the buildings. They mentioned that there is no comprehensive relationship between this existing gap and new design techniques or technologies. In other words, it is the people and not the buildings who are wasting energy (Page, 2007). The energy consumption of a building depends on a variety of factors, including the thermal and physical properties of building elements as well as its technical details. 
Meanwhile, geographic location, the way to maintain ventilation systems, and the behavior and activities of residents also play an important role (Chen, 2015, Athens, 2009). Nevertheless, understanding social aspects are more difficult than others and is referred to as "External Influences" (Athens, 2009). For example, a study suggests that the tendency of air produced by ventilation units is addictive for the residents. In the other words, the air conditioner system drives the body to hate heat. This means that the origins of air conditioning systems have been shaped on the basis of specific culture and technical standards, rather than proper scientific approaches (Prins, 1992).

Designs and constructions are rapidly moving towards sustainability and related concepts. The building industry has been revolutionized with a sustainable development approach. One of the most important parts of this movement is to set up different sets of green building ratings in the format of standards and certificates. LEED (Leadership in Energy and Environmental Design), BREEAM UK (Building Research Establishment Environmental Assessment Method) and GBL China (Green Building Label), are among these certifications. (Gou, 2017) In the United States, the nationally accepted standard for a sustainable building design, known as the LEED, is a green building valuation system developed by a National Sustainable Design Agency as USGBC, the U.S. Green Building Council, is founded in 1993 by a leading group in the North American construction industry. Basically, the LEED valuation system puts in place scores that are consistent with the satisfactory level of green building standards that are categorized into five main criteria: sustainable sites, efficient water resources, atmosphere and energy, materials and resources, and the quality of the indoor environment. Sustainability for each project is rated by the total score of these 5 points. From Duffy's point of view, LEED is a step-by-step approach to rate green buildings as a generally accepted standard for the design, construction and operation of high-performance green buildings. Duffy believes that these methodologies cannot be relied upon to stimulate strategic insights into the environmental specifications and physical features that matter most under specific circumstances (Haji Esmaili, 2011, Duffy, 2008). As Athens shows in his study of the Seattle Central Library, only 20 criteria out of the 69 criteria considered in LEED, are directly in favor of the public and 16 criteria are related to people and human behaviors (Athens, 2009).

In general, by considering different factors, designers try to estimate the energy consumption of the designed project. They implement basic design-based criteria according to existing knowledge and experience. However, numerous studies show that there is a significant difference between estimated and actual energy consumption. The actual energy consumption of the building is occasionally three times higher than the calculation (Fabi, 2013). This difference, at first glance, seems to be the result of the difference between the design of the building and its practical implementation, as well as the selection of materials and equipment and their technical and installations performance. However, in many cases, the role of individuals in the simulation of energy is not considered (Fabi, 2013, Calì, 2016). The ROWNER was a study in order to evaluate the energy consumption of buildings after occupation by residents. The analysis carried out in this study showed a significant difference between the total energy consumption of two apartments due to different behaviors of their inhabitants, such as the duration which each individual stay at the apartment, the occupancy of different spaces and the thermal preferences of individuals (HUB ZC, 2015). The behavior of the occupants of the building causes $51 \%$ change in the amount of heat, $37 \%$ 
Zargar et al., Adv. J Social Sci.; Vol. 6 Issue 1, pp: 109-121, 2020

in terms of electricity consumption and $11 \%$ in the amount of water consumption. (M. Gill, 2010) Carmalt and Wener argue that the sensitivity or sustainability of a building in response to user behavior plays an important role in determining the ability of a building to achieve its goals over its lifetime. Individuals make choices that directly or indirectly affect building performance. Measures such as adjusting the thermostat for maximum comfort of each person, adjusting curtains or opening or closing windows are among these measures (Carmalt, 2006). In January 2011, a research was conducted by Harvard University Campus Services to evalute the real amount of energy consumption in the existing bulding. In the part of their research they conducted that the occupied buildings were concuming 30\% more energy than the predicted model. (Harvard, 2018) As shown in Figure 1, the human impact on the energy consumption of buildings is related to a series of factors. Regarding this issue, this research tries to study the individuals' behavior in these environments with the aim of reaching the optimal energy consumption and the comfort of the inhabitants.

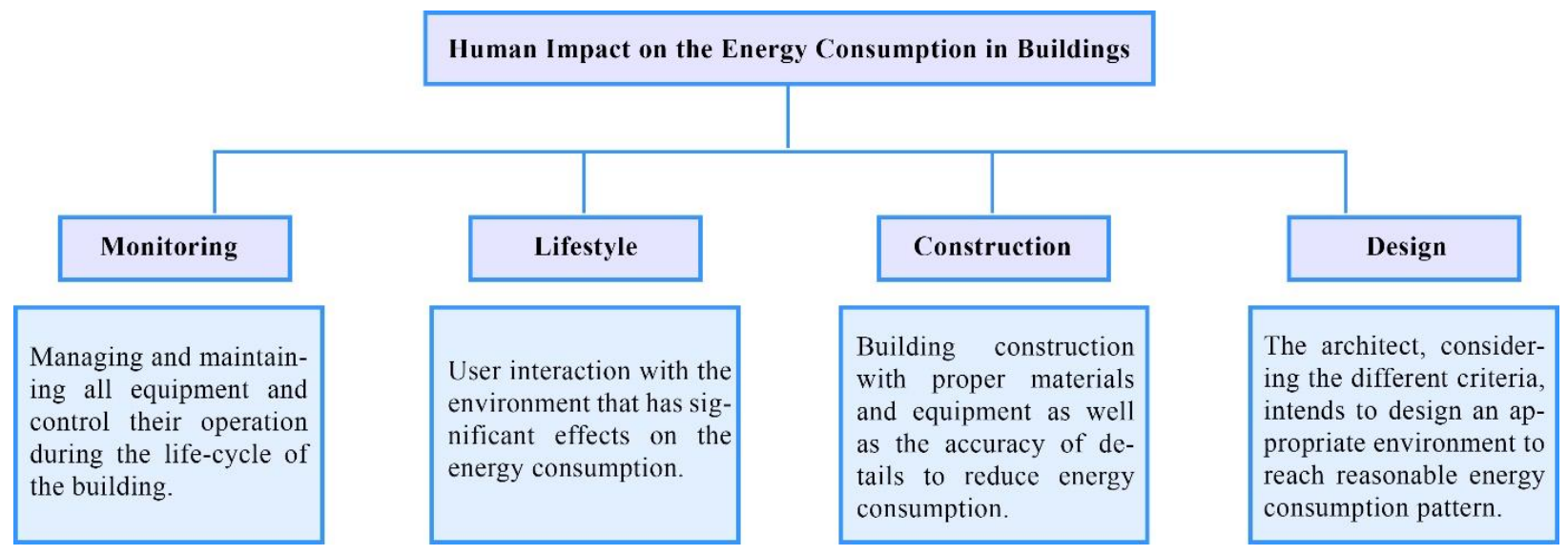

Figure 1: Human impact on energy consumption in buildings

Moreover, the social culture and beliefs are among most important factors affecting energy consumption in buildings. On the other hand, individual mistakes often cause energy dissipation in many sectors and should not be ignored in designing process. Occupants and office workers typically work for extra hours which would increase internal heat consumption due to the use of electrical and lighting equipment as well as the increase in construction services such as heating, ventilation and so on. (Sun, 2014) However, all residents do not use the same amount of facilities in a building and the role of users in energy consumption is not the same. Using computer simulations, the researchers concluded that low-skilled workers in the office environment have on average consumption of twice as high as typical and hard-working employees (Lin, 2013). In other project, power consumption after office hours at the schools and medical buildings in California, Pennsylvania and Georgia has been studied. The results indicate that the power outage rate for most equipment is less than $50 \%$, which indicates a significant potential for energy savings which is the result of the change in patterns of consumption of different individuals (Webber, 2006). Research shows that half of the individuals' workplaces are empty even at the busy days of work, and many offices use more than half of their capacity for just one-third of their daily work (N’Famory, 2018). Masoso and Grobler confirmed that a significant amount of energy is wasted during the hours of inactivity of the buildings. They tested South African buildings and Bosnia and concluded that 56 percent of the Building energy 
consumption was consumed during its passive hours (Masoso, 2010).

Building managers and people who monitor the proper functioning of equipment play an important role in energy optimization too. In addition to correct controling pattern of equipment, there are some circumstances which emerge after the introducing the new technologies or advanced systems. In these cases, people do not have the ability and training to adapt themselves to the new complexities. So, this might cause inadequate and non-optimal performance of the the proposed systems. In various cases, when a new technology is used in a building, operators and users have multiple problems in understanding the control systems and how it works. This problem will hinder the level of energy consumption to reach its standard level. Using the data provided by the New Buildings Institute and the American Green Building Association, Newsham conducted an analysis of energy use in 100 LEED-certified commercial and institutional buildings. The results show that, on average, 18-39\% of LEED buildings use less energy than similar contemporary buildings in each class, while the remaining $28-35 \%$ of these buildings have a higher energy consumption compared to ones that are similar (Newsham, 2009). These studies clearly show that, in many cases, LEED criteria did not function properly. Scofield examines energy consumption and greenhouse gas emissions for 953 office buildings in New York, which 21 of them were LEED-certified buildings, providing an opportunity for comparisons between buildings certified by LEED and other existing buildings. The results showed that LEED-certified buildings had no significant energy savings compared to other buildings. (Scofield, 2013) In general, there is a difference between the goals set for standards and what actually happens, which cause big concern and controversy over the true performance of green buildings. In many cases, buildings that are designed with the slogan of sustainable development and environmental sustainability, with a low energy consumption approach, fails to achieve the set goals in reality.

Until now, corresponding to above-mentioned paragraphs, studies in this area have generally been experimental, and are less likely to lead to introducing a functional system. So, in this research the author introduces a new human-centered approach called "Integrated Interactive System"(or in short IIS) in order to demonstrate the role of individuals in controlling energy consumption. Then, by compiling an appropriate questionnaire in Tehran, an exemplified survey about the implementation of IIS is conducted. In general, the survey tries to find practical solution for eliminating the gap between actual and expected amount of energy consumption. It is anticipated that evaluation of the overall conclusion will lead to evidence-based optimization process in the future.

\section{Integrated Interactive System}

\subsection{Background}

Integrated Interactive System (or in short IIS) performs automated boosting functions, provides userfriendly suggestions or legally enforced functions. This system interacts with the environment, individuals and energy-related equipment in order to make a suitable decision regarding different criteria. It gives suggestions to the user in accordance with the pattern of consumption. It can monitor the energy consumption of each building in various aspects, and finally it will propose practical suggestion in early stage of a design project. In addition, it can have indirect, continuous and ongoing training for residents. 
Zargar et al., Adv. J Social Sci.; Vol. 6 Issue 1, pp: 109-121, 2020

Buildings are designed to be used for a specific period of time by people from different age groups and level of awareness. There is no doubt that the proper educational planning is the best way to correct the pattern of energy consumption in a society. However, nowadays, many people in the community have no proper information about the way building systems work and how they consume energy themselves. Consequently, designing a building with the idea that all residents follow an acceptable consumption pattern is a wrong assumption. Energy-saving solutions should be adopted in such a way to reduce energy consumption over the lifetime of the building. This criterion forms IIS foundation as a human-centered approach to modify the pattern of consumption. IIS has five main suggestion patterns based on a datadriven process:

\subsubsection{Absolute Law (Legal Controls)}

The simplest possible way is legal action. By enforcing the law and strict implementations, the goal is to confront any problems or disorder in consumption pattern. An example of law enforcement is the quota of household water in some cities and countries. (Jiang, 2010) Each household has a maximum proportion of consumption and people in the community cannot use more than the quota even in return for higher price.

\subsubsection{Law and Quota Steps}

This approach comes with law enforcement along with the allocation of quotas individually. The energy consumption is divided into different steps, and for each range of consumption, different and incremental financial costs are measured. With increasing consumption of each person, the payment also rises with a proper increasing pattern. In contraction to "Absolute Law", the user, who exceed the consumption limit, is capbale to consume energy but in a higher price.

\subsubsection{The Punitive Law}

This step is similar to the two methods mentioned above, except that it does not deal seriously with severe consumption. It will consider non-monetary fines for people with an unusual pattern of consumption. In this approach, excessive consumption would result in fines in the same area of energy consumption patterns or other areas. The reduction of water pressure is among these measurements.

\subsubsection{Legal-functional training}

This training will lead to the correct implementation of the law in a culture-based approach. In this scenario, the rules will be clarified to the community. The upcoming punishments and penalties resulting from violations of these laws will be taught too. The overall goal of this training is to modify the consumption pattern of each household in accordance with the statutory requirements. The implementation is directly related to the proper definition of the infrastructure designated for energy consumption (building facilities). Part of this training includes text warnings (such as bills for families with high consumption) or other visual warning equipment implemented as a part of buildings' main facilities. As mentioned, the decision-markers tries to modify consumption pattern in a friendly environemet; so, no legal restrictions will apply. 


\subsubsection{Emotional training}

This training focuses on the emotional aspects which makes people investigate their own consumption pattern flaws. Comparing the consumption of each household with similar households or video tutorials such as making documentaries and teasers are among these trainings.

\subsubsection{Basic Education}

Basic training is the foundation of a true consumption pattern. This training requires a precise and longterm plan. The goal is to prepare children in educational environments or people in the community to rebuild and modify their consumption pattern in a step by step process. Obviously, the correct implementation of IIS depends on many environmental, individual and even legal factors. IIS suggests this approach in the specific "Ideal" conditions to reduce consumption. The proper implementation of the idea varies according to related criteria such as education, culture, the time of construction, site-plan etc. (Figure 2). The information can be extracted in various form of data. For instant, this research uses a questionnaire approach to collect necessary data from a real case study in Tehran. After data evaluation, it leads to best suggestion pattern using IIS.

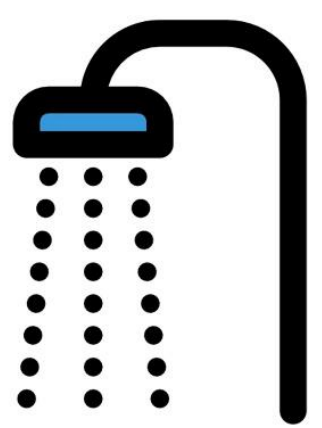

A resident is getting a shower at a specefic time of the day. After several minutes, the amount of water consumption will exceed its limit
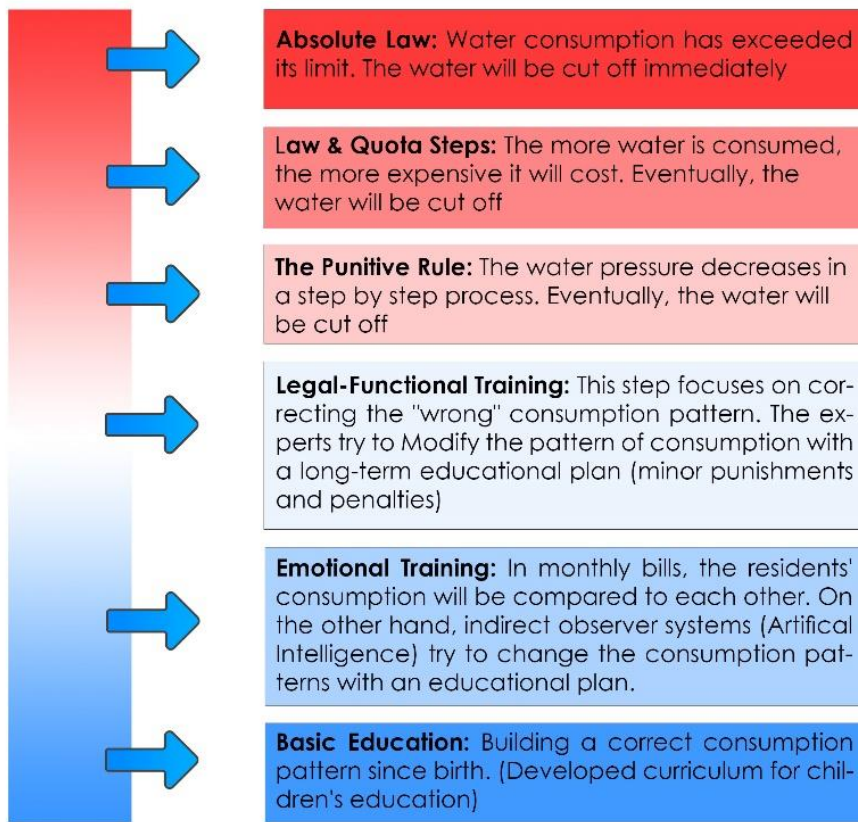

Law \& Quota Steps: The more water is consumed, the more expensive it will cost. Eventually, the water will be cut off

The Punitive Rule: The water pressure decreases in a step by step process. Eventually, the water will be cut off

Legal-Functional Training: This step focuses on correcting the "wrong" consumption pattern. The experts try to Modify the pattern of consumption with a long-term educational plan (minor punishments and penalties)

Emotional Training: In monthly bills, the residents' consumption will be compared to each other. On the other hand, indirect observer systems (Artifical Intelligence) try to change the consumption patterns with an educational plan.

\section{Basic Education: Building a correct consumption} pattern since birth. (Developed curriculum for children's education)

Figure 2: The range of IIS choices when there is a mistake in the pattern of consumption.

\subsection{IIS Featurs: primary information}

\subsubsection{The Role of Social Behavior}

In many post-occupancy studies, desingers will give some information to typical residents about how the building is designed with the engineering perspective, why some special facilities are choosen for a project or even why the bathroom is located in specific place. Although the need for a scientific or executive specialist to advance the design process is inevitable, these are users who have the main role in redesigning the internal space and creating environmental amenities. Due to that reason, paying attention to the resident's view is worthful and must be taken into account in all stages of the design. IIS pays special 
Zargar et al., Adv. J Social Sci.; Vol. 6 Issue 1, pp: 109-121, 2020

attention to users during the lifecycle of the building, so the output is based on direct interaction with the residents.

\subsubsection{Learning and Adaptation}

User interaction with building systems can lead to proper learning too. IIS could be smart by storing information over time and making adaptation in future decisions. This learning takes place in various areas, like the period of climate fluctuation or changes in people's view (such as aging, illness etc). The learning process can be done in a one-dimensional process, (interaction of each separate system with its environment) or in a multidimensional and cumulative process (conjunction with information obtained from systems which are used in same situation all around the world).

\subsubsection{Cultural Practice}

As it is mentioned before, many human factors have impacts on the energy consumption of a building. It is clear that the consumer needs to be guided by an invisible hand to put him in the right direction. However, the invisible hand does not mean creating new problems for the residents. IIS must work with comprehensive and well-defined criteria. So, by using the outcupts in a proper and accurate way, the defined system can reach the main human-centered goal. Regrading this issue, the cultural practice can be used in order to spread the concepts of energy consumption through videos or games or in the context of the rules and regulations. However, some rules are not acceptable by the residents in the first place. The decisionmakers who choose the legal actions must pay close attention to the culture of the residents. In some cases, such actions may cause problems in the community.

\section{Results}

The selection of each action and reaction in IIS is dependent on several factors. Geographic location, cultural background, infrastructures, trust in government actions, financial resources, as well as social behaviors, all contribute to proper responding of the IIS to its surroundings. These are the factors that determine residents' feedback to the architect's design. In order to explain one of the methods for obtaining initial information about residents' behavior and thoughts, a questionnaire was prepared based on Likert criteria. The survey has been developed in 37 questions and is conducted among people living in Tehran metropolitan area, searching for an acceptable and suitable solution for designing a typical residential building in the Tehran's downtown. In general, the approach is defined as follows:

1. At the first step, by studying the participants lifestyle, culture and education, a proper view is reached by authors to answer "What are the main implementation priorities?"; In other words, for useful IIS operation in Tehran, "What priorities should be given to various issues related to energy consumption such as heat, light, water, etc.?”

2. Implementing IIS will certainly have many positive and negative feedbacks. The compilation questionnaire answers "How people will respond to these changes?", "Do residents have a positive approach regarding the defined system?", "Are there differences between the expert's perspective (engineers, architects, etc) and common occupants in this area?", and finally, "How far can the designers go toward the main goals of IIS?". 
3. Regulations such as LEED are essential for designing construction projects. Besides that, there are laws, requirements and other constraints on the energy saving approach that employers, contractors and building users must comply. In the questionnaire, some questions are raised which examines the effectiveness of these rules and then evaluate the people's response to these rules. The output will help authors to shape the IIS approach more rigorously.

Four questions of the survey are about gender, age, education, and the level of acquaintance of each participant with the fields of civil, architectural and environmental engineering. In 33 remaining questions, information is collected based on Tehran's energy consumption pattern. Answering the questions is based on a Likert scale, a psychometric scale, which is frequently used in research questionnaires. This scale is one of the most reliable methods used to measure people's attitudes or behavior. Likert scale Measurements of behavior and attitudes are evaluated using multi-choice responses that range from a low to high limit (for example, I totally disagree to fully agree). Options such as 'I do not agree' or 'disagree', 'I'm not sure', 'as I expected' and 'I have no idea' are usually used for the middle option of the spectrum. Opposite to simple "yes/no" questions, the Likert scale has the potential to reveal the points of view, which can be useful especially for sensitive and challenging subjects, and the ability to have a better understanding of trends tending to be available in a range of responses. Usually, options are not valued in order to prevent respondents to be influenced by numbers. In this questionnaire, evaluative options are designed according to table 1:

Table 1: Valuation of answers to questions in the five-point Likert range

\begin{tabular}{|c|c|c|c|c|}
\hline Totally Disagree & Disagree & No Idea & Agree & Totally Agree \\
\hline-2 & -1 & 0 & 1 & 2 \\
\hline
\end{tabular}

In this research, the number of participants in the questionnaire is 60 , of which $46.7 \%$ are men and $53.3 \%$ are women, and $53.3 \%$ of all participants have education in civil, architecture or urban fields. All information extracted from the questionnaire was stored in charts and tables for further analyses (Figure 3). The questions are divided into three groups (related to the behavior of the building users), (macro issues related to design criteria), and (issues determining how the IIS works correctly).

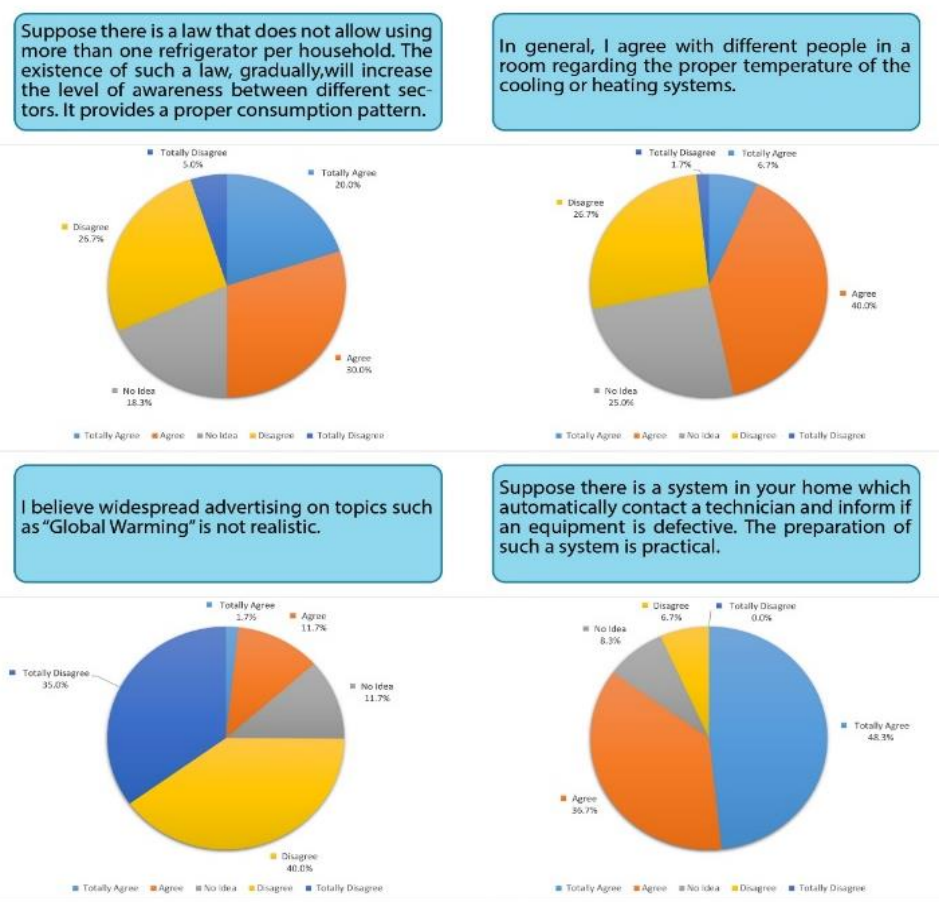

Figure 3: Circular diagrams of preliminary results extracted from the questionnaire 


\section{Discussion}

The infrastructure of the IIS offers actions to modify consumption pattern. In Figure 4, the range of IIS choices is shown according to the valuation of the questionnaire. In this section, the IIS scoring system is evaluated based on different variables. The output of this assessment system is applicable in planning, design and construction phases.

\subsection{Building Design}

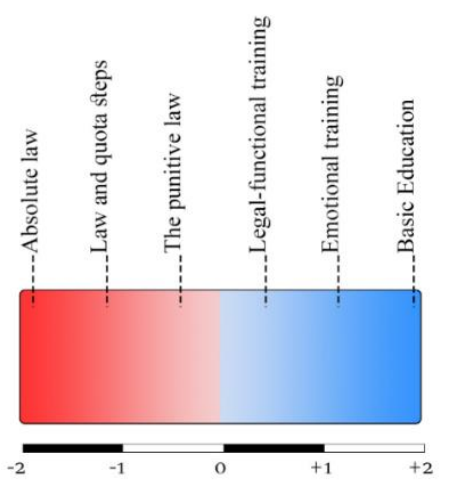

This section is related to the issues that the residents inevitably faced with; For example, the fluctuations of the building's heating system temperature, or the coldness of the bathroom. The survey is designed to measure the level of participants' satisfaction in residential buildings. According to the results of the survey (Figure 5), the average score of residents according to these criteria is below zero, but it is not very critical due to its balance and intermediate mode. It seems that paying attention to legal measures in the modern buildings design is inevitable in Iran. Therefore, during design and construction, special consideration should be given to issues such as appointing responsible technicians for the installations, realization of the rules and the

Figure 4: The range of IIS choices according to the valuation of the information

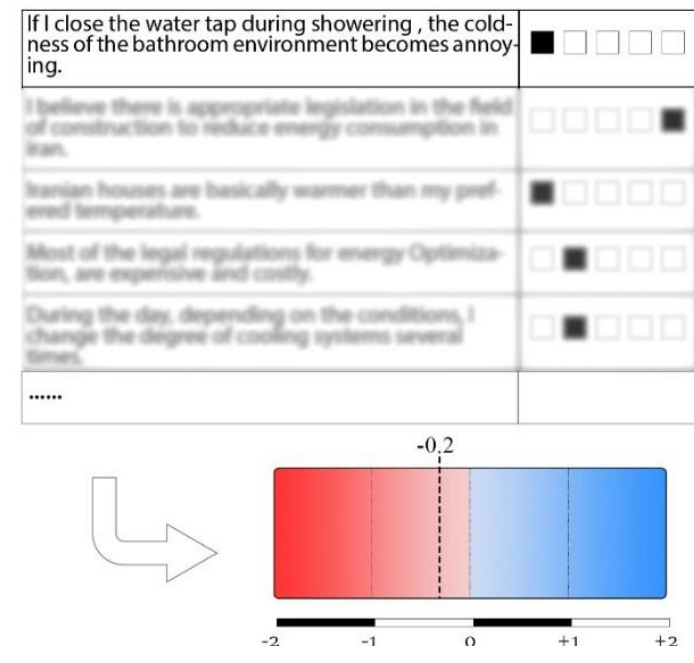

Figure 5: Major questions related to building design next to the range of IIS choices. implementation of the defined principles such as energy standards. However, the construction status in the energy-related areas is roughly acceptable. According to the results, in most cases, the reasonable progress of the construction industry in Iran has made the building users satisfied with the general condition of their home. However, extracted data indicates that some people with specific tastes that are not happy with today's trend of energy-based construction in Tehran. The sum of these data sets us in the middle of the IIS chart in this section.

\subsection{Building Users}

In this case, the building is assumed to have the best design criteria. However, the residents have special habits and lifestyle (leaving the windows open, taking a long shower, etc.), which cause problems in the optimal energy consumption pattern. The general purpose of this section is to create an overview for designers about the specific and influential behavior of residents in energy consumption. As shown in Figure 6, the results tend to be in the blue section. It can be concluded that, in recent years, the training of the correct pattern of consumption has led to an increase in the level of information of individuals. However, the existence of an average of 0.34 is not so reliable and the necessity of progressing in the field 
Human-centered Approach for Proper Functioning of LEED Criteria; A Case Study in Tehran

of education is evident. It can be concluded that in these cases, it is possible to guide the residents in the proper way of using the equipment by occasional training.

\subsection{IIS (Sample Case)}

In this section, a specific part of the IIS has been investigated. This part is defined directly according to the criteria mentioned in Section 2. By interacting with the daily lives of residents, IIS tries to correct their consumption pattern. Figure 2 showed the range of IIS choices when there is a mistake in the pattern of consumption. Given this, an observer system was defined, and its proper functioning is evaluated by the participants. The system was designed to observe the behavior of living users indirectly and to make the necessary decisions. Here are two sample questions which evaluated based on system's specification:

1. Suppose a device is designed to alert you when you use too much water in the bathroom, and if you continue using water, it will reduce the pressure of the water. I think that the existence of such a device will help to modify the pattern of consumption over time.

2. Suppose there is a special thermal camera installed in your home and a computer software controls the temperature of the home environment by analyzing traffic and activity. The existence of such a camera violates residents' privacy.

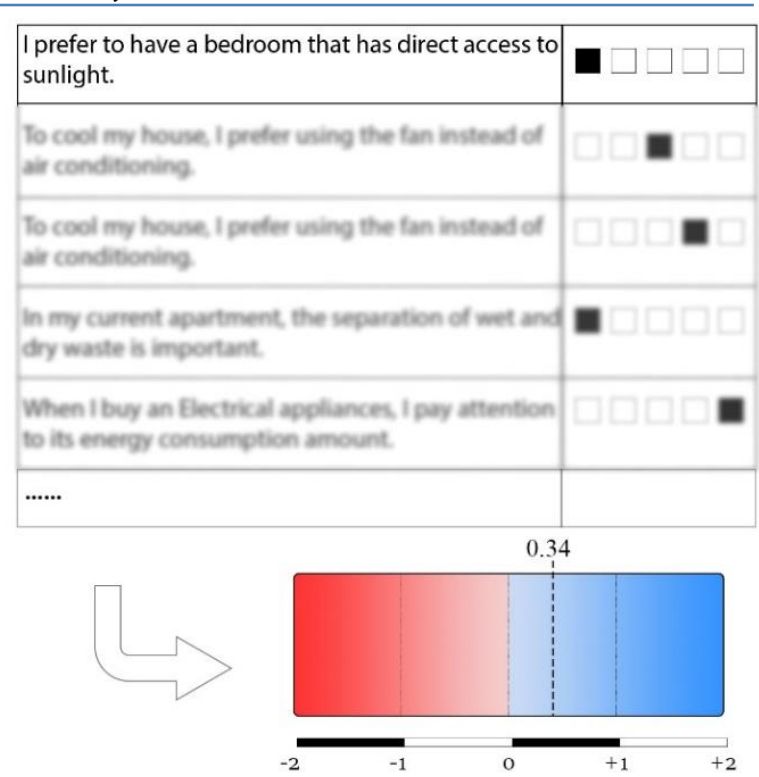

Figure 6: Questions related to behavior of Building users next to the range of IIS choices

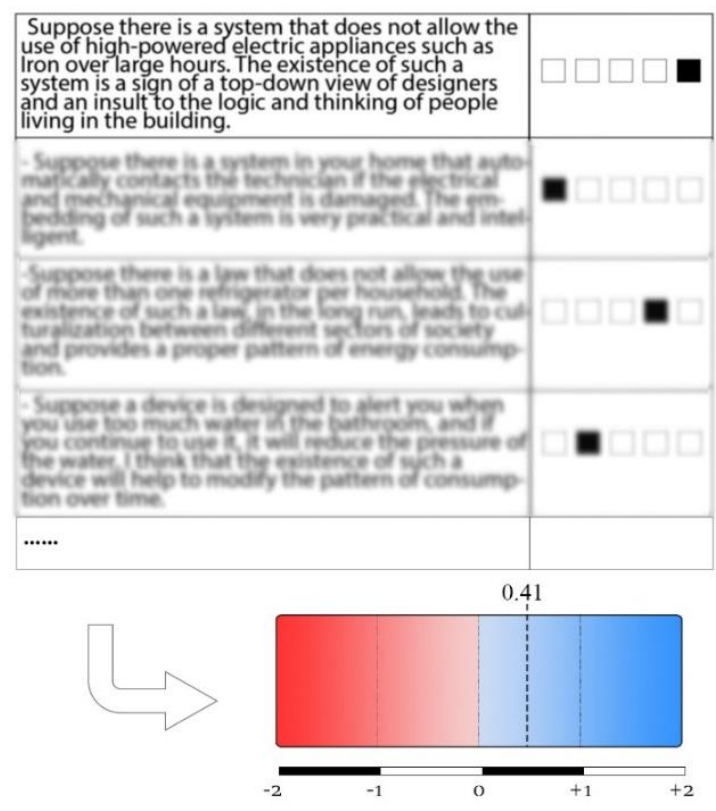

Figure 7: Determinant questions of the IIS correct functioning next to the range of IIS choices

As shown in Figure 7, the result is positive and indicates that residents have no major problem with such an observer system. Yet, since the score of this section is close to zero, it is necessary to have proper planning options for getting the residents' decisive consent. The design of such a system can be done with an extra cost and specific hi-tech equipment. According to the positive attitude of participants, the guidance and accompaniment of people will help the engineers to focus on the design and implementation of these technologies. In the next step, special workshops must be held to indicate the positive and negative aspects of propsed systems. 
Zargar et al., Adv. J Social Sci.; Vol. 6 Issue 1, pp: 109-121, 2020

\subsection{Energy consumption}

The survey can be divided into two different parts; "Light and Electricity" and "Sense of Environment". As shown in Figure 8, the results are in the positive parts. Although there is not a substantial conclusion from these results, this indicated that long-term social media advertisements can be one of the important factors influencing public opinion in order to modify the pattern of consumption in a long period of evaluation.

Moreover, it can be rendered that there is a tide connection between people's previous knowledge in the related fields and the amount of consumption. Most of the participants were not well aware of the correct consumption pattern but they are willing to learn more. For instant, most of the contributors in Tehran believe that there is a rigorous connection between electricity consumption and appliances efficiency. However, it is evaluated from survey that they do not pay much attention to electrical appliances' energy ratings when they decide to buy one. Consequently, their beliefs will not have a practical or intuitive aspect.
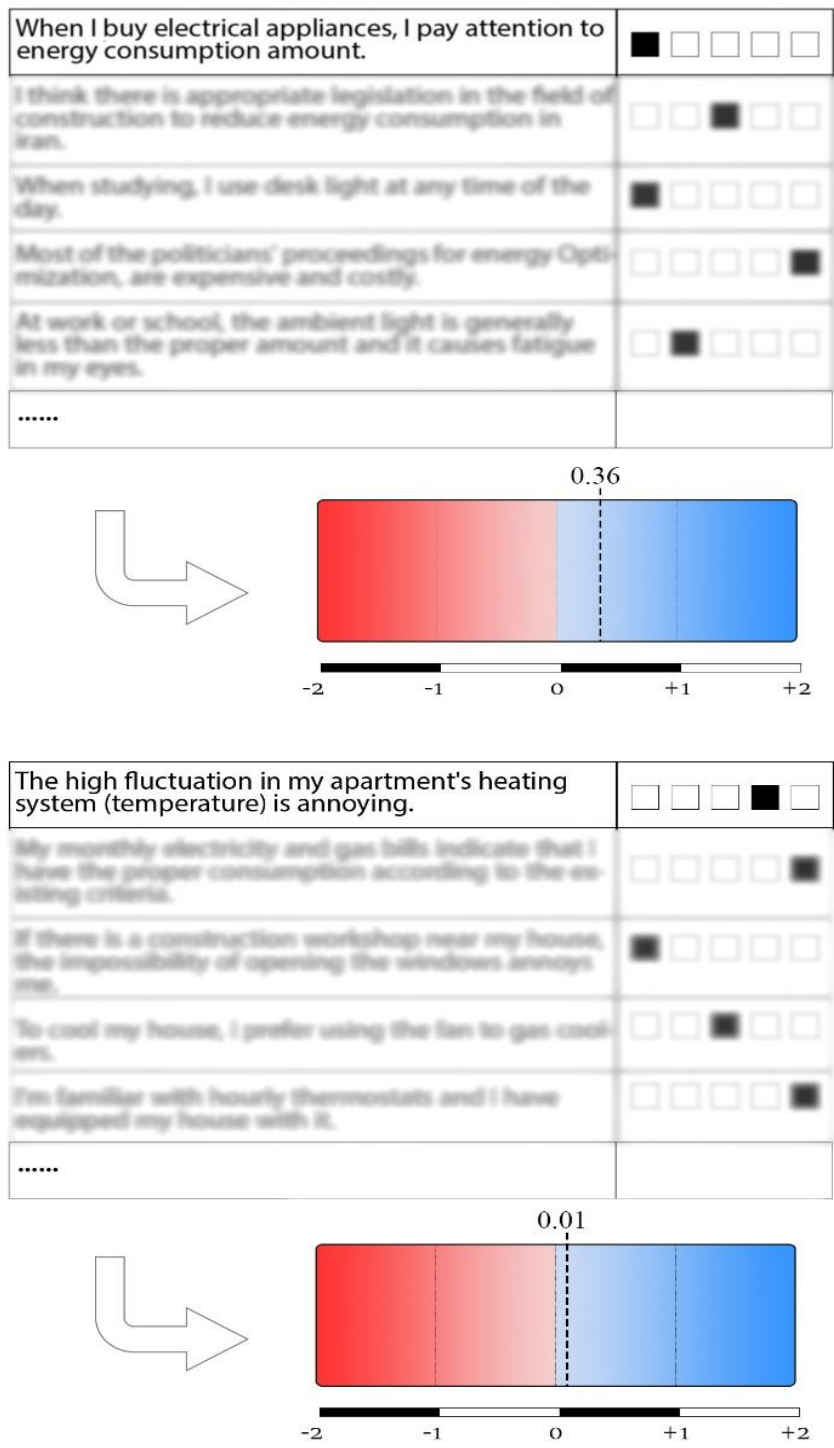

Figure 8: (Up: "Light and Electricity" evaluation) and (Down: "Sense of environment" evaluation) next to the range of IIS choices

\section{Conclusion}

This research introduced Integrated Interactive System (IIS) as a human-centered approach that interacts with the environment, individuals and energy-related equipment. It performs auto-optimization actions, provides user-friendly suggestions, or legislate legally enforced functions. It can monitor the energy consumption of each building in a variety of areas to correct consumption patterns. In addition, the system is capable of undertaking necessary legal measures and indirect, continuous and ongoing training for residents. Choosing different approaches to the system requires a preliminary study by the design team to find out about the social behavior and beliefs of the users. The best way to extract such information is to involve the residents in the design process. Moreover, it is beyond doubt that the complete awareness of designers about social lifestyle plays a significant role in reducing energy consumption during the operation of the building. In this research, a survey was conducted among Tehran's residents. By using the outputs, the authors properly evaluate part of their suggested system. The extracted information represents the noteworthy participants' knowledge in the field of energy consumption. However, they need to update their 
Human-centered Approach for Proper Functioning of LEED Criteria; A Case Study in Tehran

training in the related area based on new technologies and enhancements. New information can be learned through government planning and advertising. Participants also believe in the important role of new and observer technologies in controlling energy consumption patterns. As a result, the designers are allowed to use indirect controller approaches to monitor energy consumption in different parts of the building using artificial intelligence. The IIS has a fixed infrastructure that can be used as the basis for all designs and construction projects. Nonetheless, there are numerous methods to evaluate these parameters for different design projects.

\section{Declarations}

\subsection{Informed Consent}

Informed consent was obtained from all individual participants included in the study.

\subsection{Funding}

This research has no funding

\subsection{Competing Interests}

The authors declare that they have no conflict of interest exist.

\section{How to Cite this Article:}

Zargar, S. H., Dehghani Firouzabadi, F., Zareie, M., \& Khaghani, S. (2019). Human-centered Approach for Proper Functioning of LEED Criteria; A Case Study in Tehran. Advanced Journal of Social Science, 6(1), 109-121. doi:10.21467/ajss.6.1.109-121

\section{References}

Athens, Lucia (2009). "Design for social sustainability at Seattle's central library”, Green buildings, V. 2:1.

Calì D, Osterhage T, Streblow R, Müller D (2016). Energy performance gap in refurbished German dwellings: lesson learned from a field test. Energy Build; 127:1146-58.

Carmalt, Hannah and Wener, Richard (2006). "Environmental psychology and sustainability in high-rise structures”, Technology in Society, V. 28, pp. 157-167.

Chen S, Yang W, Yoshino H, Levine MD, Newhouse K, Hinge A (2015). Definition of occupant behavior in residential buildings and its application to behavior analysis in case studies. Energy Build;104:1-13.

D’Oca s, Hong t, Langevin J (2018). The human dimensions of energy use in buildings: A review. Renewable and Sustainable Energy Reviews $81 ; 731-74$.

Duffy, Frank (2008). Work and the city, Black dog publishing.

Fabi V, Andersen RV, Corgnati SP, Olesen BW (2013). A methodology for modelling energy-related human behaviour: application to window opening behaviour in residential buildings. Build Simul ;6:415-27.

Gou, Z.; Xie, X (2017). Evolving green building: Triple bottom line or regenerative design? J. Clean. Prod., 153, 600-607.

Haji Esmaili, Yasaman (2011). Consideration Of Human Behavior In Designing A. Green Office Space For Environmentalists. A Simulation Study. A Master's Report Submitted to the Faculty of the Department of Architecture, Graduate College of The University of Arizona. Harvard (2018), Green is the new Crimson, Green Building Service, Harvard University.

HUB ZC (2015). Post-occupancy Evaluation, Rowner Research Project Phase Two. Zero Carbon HUB.

Janda KB (2011). Buildings don't use energy: people do. Archit Sci Rev;54:15-22.

Jiang Y, Chen Y, Younos T, Huang H, He J. Urban water resources quota management: the core strategy for water demand management in 
China. Ambio. 2010;39(7):467-75.

Lin HW, Hong T (2013). On variations of space-heating energy use in office buildings. Appl Energy;111:515-28.

M.Gill, Zachary and Tierney, Michael J. (2010). "Low-energy dwellings: the contribution of behaviors to actual performance”, Building

Research and In $\neg$ formation, V. 38(5), pp. 491 - 508.

Masoso, O. T., and Grobler, L. J. (2010). The Dark Side of Occupants' Behaviour on Building Energy Use. Energy and Buildings, 42(2), 173177.

N'Famory Camara, Deyi Xu, Emmanuel Binyet (2018). Enhancing household energy consumption: How should it be done? Renewable and Sustainable Energy Reviews 81; 669-681.

Newsham, G.R.; Mancini, S.; Birt, B.J (2009). Do LEED-certified buildings save energy? Yes, but . . . Energy Build. 41, 897-905.

Page J, Robinson D, Scartezzini J-L (2007). Stochastic simulation of occupant presence and behaviour in buildings. in: (LESO-PB) SEaBPL, editor. CH-1015 Lausanne, Switzerland Ecole Polytechnique Fédérale de Lausanne (EPFL).

Prins, Gwyn (1992). “On Condis and Coolth”, Energy and Buildings, V. 18, pp. 251 - 258.

Scofield, J.H (2013). Efficacy of LEED-certification in reducing energy consumption and greenhouse gas emission for large New York City office buildings. Energy Build. 67, 517-524.

Sun K, Yan D, Hong T, Guo S (2014). Stochastic modeling of overtime occupancy and its application in building energy simulation and calibration. Build Environ; 79:1-12.

Webber, C., Roberson, J., Mcwhinney, M., Brown, R., Pinckard, M., and Busch, J (2006). After-hours power status of office equipment in the USA. Energy, 31 (14), 2823-2838.

Publish your research article in AIJR journals-

$\checkmark \quad$ Online Submission and Tracking

$\checkmark$ Peer-Reviewed

$\checkmark$ Rapid decision

$\checkmark \quad$ Immediate Publication after acceptance

$\checkmark$ Articles freely available online

$\checkmark \quad$ Retain full copyright of your article.

Submit your article at journals.aijr.in

\section{Publish your books with AIJR publisher-}

$\checkmark \quad$ Publish with ISBN and DOI.

$\checkmark$ Publish Thesis/Dissertation as Monograph.

$\checkmark$ Publish Book Monograph.

$\checkmark$ Publish Edited Volume/ Book.

$\checkmark$ Publish Conference Proceedings

Retain full copyright of your books.

Submit your manuscript at books.aijr.org 